\title{
Dynamic Hardware-in-the-loop UAV Ground Testing System
}

\author{
V.M. Sineglazov \\ Computer-Integrated Complexes Department \\ National Aviation University \\ Kyiv, Ukraine \\ svm@nau.edu.ua
}

\author{
S.O. Dolgorukov \\ Computer-Integrated Complexes Department \\ National Aviation University \\ Kyiv, Ukraine \\ sdolgorukov@nau.edu.ua
}

\begin{abstract}
The paper shows how dynamic hardware-in-the-loop ground test system can be used when solving problems for preflight testing in unmanned aerial vehicle development process. The problem of different unmanned aerial vehicle subsystems check of different-type features for irregularities discovery and decisionmaking during extensive test procedures is considered. The paper also describes the architecture of a developed dynamic test rig that can be used for test program data acquisition and analysis.
\end{abstract}

Keywords-ground testing; hardware-in-the-loop; unmanned aerial vehicle; testing system

\section{INTRODUCTION}

Unmanned aerial vehicle (UAV) embedded control systems - UAV ECSs - are becoming more widespread in aviation recently. Compared to usual flight computer programming implementations they have added problems like robustness, code efficiency, code quality and safety which make them difficult to implement and highly error-prone. Moreover, such systems are used in critical and hazardous UAV applications, where a precise and methodological implementation is mandatory. Most ECSs are therefore very conservative in their implementation and the development costs are much bigger than for usual computer programs. Moreover, it is difficult to replace or correct software errors after shipping, and consequently the ability to test such systems methodologically before shipping is very important.

\section{UAV SYSTEMS RELIABILITY}

Hardware-in-the-loop system for ground testing can be implemented in many different ways. The implementation strategy is usually a project and resource dependent matter. To discuss this we use the categorization given by Isermann in [6]. As can be seen from Table I not all the combinations are feasible, more precisely configurations 3,7 and 8 are impossible, since a simulated actuator which deliver a physical output is a real actuator of the system, and similarly a simulated sensor which reads physical inputs is a real sensor. Configuration 4 is the real working system, since no simulation is involved, whereas 5 is a fully simulated system. Such a configuration is called Full Simulation. When areal component is in the simulation loop it is called Partial Simulation.

Note that during the development cycle we are able to move from configuration 5 to 4 replacing real actuators, real sensors and eventually the real process. These transitions however are very difficult to implement since at each step a new physical interface has to be implemented between simulation and real actuator / sensor.

TABLE I. HARDWARE-IN-THE-LOOP SYSTEM FOR GROUND TESTING

\begin{tabular}{|c|c|c|c|c|c|c|c|}
\hline \multirow{2}{*}{ Case } & \multicolumn{2}{|c|}{ Actuators } & \multicolumn{2}{c|}{ Process } & \multicolumn{2}{c|}{ Sensors } & \multirow{2}{*}{} \\
\cline { 2 - 8 } & Real & Sim. & Real & Sim. & Real & Sim. & \\
\hline 1. & + & & & + & & + & \\
\hline 2. & + & & & + & + & & \\
\hline 3. & + & & + & & & + & Impossible \\
\hline 4. & + & & + & & + & & $\begin{array}{c}\text { Real } \\
\text { System }\end{array}$ \\
\hline 5. & & + & & + & & + & $\begin{array}{c}\text { Full } \\
\text { Simulation }\end{array}$ \\
\hline 6. & & + & & + & + & & \\
\hline 7. & & + & + & & & + & Impossible \\
\hline 8. & & + & + & & + & & Impossible \\
\hline
\end{tabular}

\section{A. Systems of $U A V$}

The overall system may be considered for convenience in two parts (Fig. 1).

1) The computing part of the system which accepts the commands from the operator (in short-term or long-term), compares the orientation, etc. of the aircraft with what is commanded, and instructs the other part of the system to make appropriate correction. This is often referred to as the automatic flight control system (AFCS) or FCS logic, and contains the memory to store mission and localized flight programs.

2) The mechatronics of the system which accept the instructions of [1] and apply input to the engine $(s)$ controls and / or aerodynamic control surfaces.

The typical AFCS may consist of the following [7].

I. Six Type of Sensors:

1) 3-axis Rate Gyros;

2) 3-axis Accelerometers;

3) absolute Pressure sensor to measure the altitude;

4) differential Pressure Sensor to measure airspeed;

5) 3-axis Magnetometers;

6) GPS. 


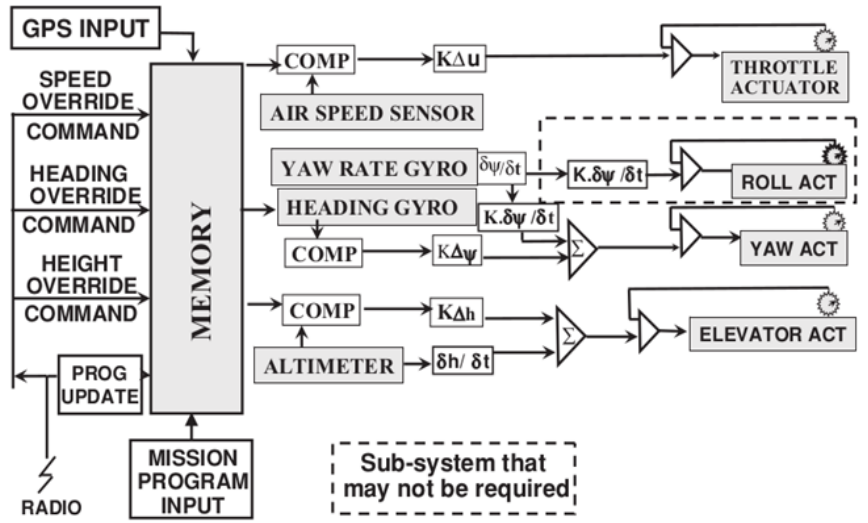

Fig. 1. Typical UAV system.

II. Flight computer that reads the data from the sensors and according to its program it will send signals to the servo motors to follow its calculated mission.

\section{Servo Motors that:}

- move the aileron to roll;

- move the elevator to pitch;

- move the rudder to yaw;

- change throttle for speed.

\section{B. Reliability considerations}

The reliability of a UAV system (Fig. 2) must be assured for the following reasons:

a) If a UAV system fails whilst on a mission, then that mission has failed. In a military operation, this lack of information, etc., could result in loss of initiative or worse, hundreds of deaths. In both military and civilian operation a failure could result in injury or even loss of life to the operators.

b) If the aircraft crashes, injuries or fatalities might be caused to the over-flown population.

c) Any loss or malfunction of the system can result in loss of the service provided, loss of the facility and costs of repair or replacement. Unreliability is a major driver in whole life costs.

The costs added to achieve a specified level of reliability depend upon:

- the complexity of the total system;

- the level of reliability specified under specified ambient conditions - temperature, altitude, humidity, precipitation, day/night, type of operation, etc.;

- the availability of components with a known level of reliability;

- the success of the design phase in 'designing-in' reliability.

Therefore it is impossible to generalise what the total costs will be. Even if a history of the development costs of previous systems is known, no method has yet been devised to use that information to predict the costs of a future system. Therefore the determination of a required level of system reliability on an economic basis does not appear to be currently feasible.

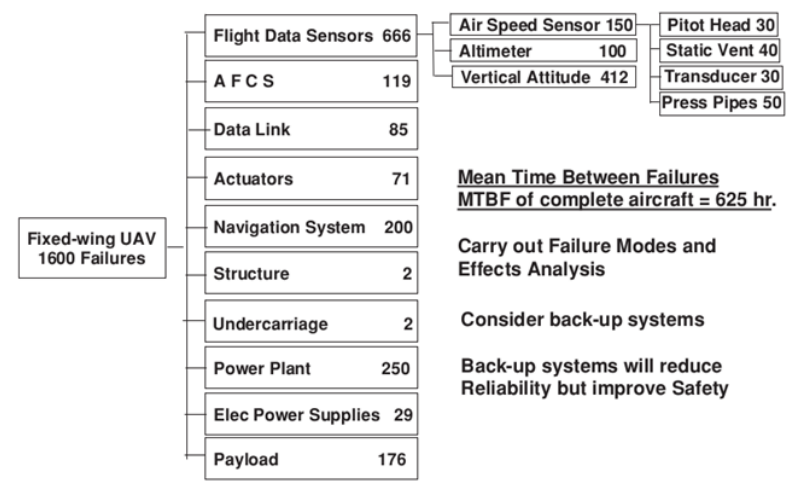

Fig. 2. Historical system reliability performance in failures per one million flight hours.

\section{UAV TESTING SYSTEM}

The pre- and post-flight checks will be carried out including checks for wear, fluid leaks, signs of overheating, security of connectors, etc. At some stage, communications testing must be carried out on a prototype UAV to prove radio transmission and reception. This will include confirmation of acceptable antennae positioning to achieve adequate gain at all UAV to control station (CS) orientations. It may therefore be necessary to mount the airframe on an elevated platform. It also becomes necessary to ground-test a complete UAV with subsystems operating.

At some time the largely complete UAV must become airborne under control from the CS. To reduce the risk attendant on that first flight, as many as possible of the subsystems will be progressively integrated into a complete airframe and tested for correct functioning and to ascertain if there is any undue adverse interaction between them. These unwanted interactions may be due to electro-magnetic interference, vibration or inter-system heating, etc.

It may be necessary to construct the ground test system or rig upon which to mount the complete UAV attached to the test system at strong-points on the fuselage or wing attachment points. An interpretation of this type is shown in Fig. 3. The rig should permit pitch, roll and heading attitude control according to the test program.

\section{Data Acquisition And Test Procedures}

Appropriate instrumentation are set up to measure and record commands, responses and conditions. The more critical values are displayed. This instrumentation include linear and/or angular potentiometers to measure control surface and throttle displacements, ammeters, voltmeters, temperature measurement, accelerometers, strain-gauges and engine speed measurement, etc. It may also be possible to include means of measuring propeller thrust which would be of particular advantage in future in-flight testing. Most of the 
instrumentation will be carried on to in-flight testing and, in addition to performing its task on the ground rigs, will be proven for the later operation.

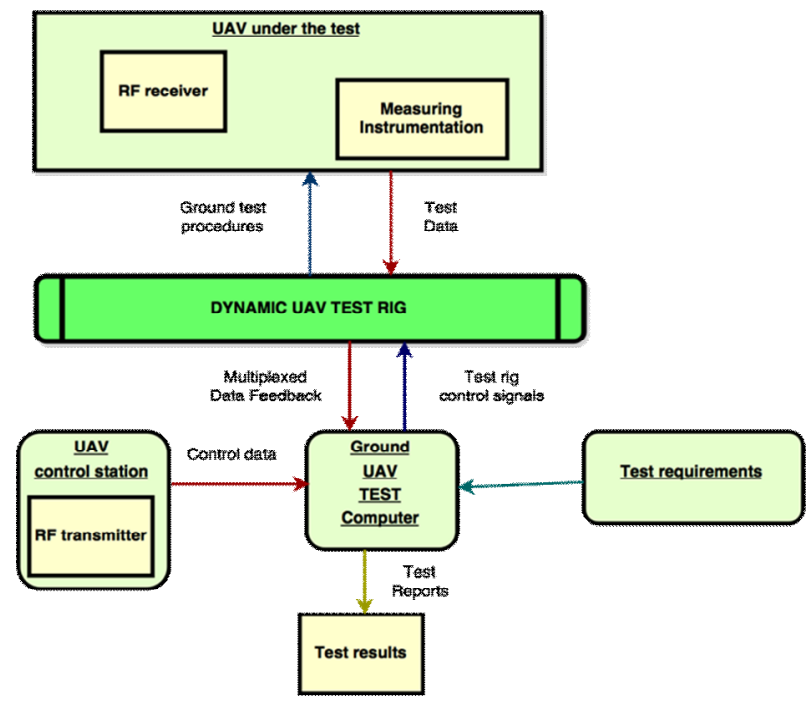

Fig. 3. UAV ground test system.

It may be considered prudent to assess the structural characteristics of the wings, in particular the torsional stiffness and position of the flexural axis in order to assure the nonoccurrence of aerodynamic flutter. Determination of the flexural axis position is often accomplished by mounting the UAV upsidedown in the rig and loading the wings with weights along the line of the aerodynamic centre, representative of the span-wise lift distribution at maximum lift. The maximum lift is that specified by the Flight Envelope in the Type Record. Measurement of wing deflection at the leading and trailing edges will enable the position of the flexural axis to be determined and, if found to be too far aft of that calculated in design, correction can be made by adding mass forward or adding suitable stiffening. It may also be opportune to measure the stresses at critical points such as the wing root fittings.

Unmanned aerial vehicle ground test system must ensure tests on the parameters close to real, namely the angular positions, overload, angular velocity and acceleration of all three control channels - pitch, roll and heading. To ensure these parameters on the technical design stage it is necessary to solve the task of developing assembly units, functional task, the task of developing algorithms and software, the task of selecting a set of technical means. The installation and functioning of the several sub-systems may be made in total from the beginning or more usually added as the program of testing is developed.

System Integration Testing (SIT) is a critical phase, which typically takes place in a lab environment following individual component and subsystem testing. In most cases this is the first time that all of the components and subsystems are exercised in the intended operational configuration. Historically, too little time and resources have been allocated for this effort as it is typically the last phase before formal Developmental Testing (DT) begins. Any schedule slippage that occurs during development usually results in compression of the time allotted for SIT. In addition, configuration management must be in place at the start of SIT, adding to the time required to implement the changes needed to fix the inevitable discrepancies that will be discovered. System Integration Testing is intended to find the problems not discovered in the traceability of functional requirements and Interface Control Documents used in the system design. These critical documents should be verified and corrected during the SIT. The SIT test set up should include the control station, Air Vehicle, Data Links, Launch and Recovery Systems, and any other subsystems required for the system to execute the mission. As the size of the air vehicle increases, it may not be practical to house the entire aircraft in a lab environment. In this case, actual aircraft hardware should be utilized to the maximum extent possible (for instance, actual control surface actuators or servos can be driven by control system commands in the SIL). Therefore in such cases all vital UAV systems should be mounted on ground test system.

Few modern UAVs operate with direct rate controls. At one time, rate control was the only mode of operation Remotely Piloted Vehicles. Attitude sensing and stabilizing systems are nearly always employed, as well as some form of inertial or GPS navigation. While these systems will most likely be tested during component and SIT, it is imperative that they be exercised immediately prior to flight testing to ensure that they are operational and that their operating sense is correct.

The attitude control system may be as elementary as a single rate gyro mounted on an incline to sense both roll and yaw, and to provide basic wing leveling. Such a system combined with a barometric sensor controlling altitude can provide basic autopilot and autonomous flight functions. More often, a vertical reference gyro with a yaw rate gyro and air data computer will be used to provide position control and autonomous operations.

Tactical and larger systems may employ redundant ring laser gyros and other attitude computing systems. Regardless of the component architecture, some basic safety of flight ground tests must be conducted. In cases where the design incorporates well-developed flight control laws, they can be assessed in terms of transfer functions to ensure that the correct control surface deflections result from measured attitude deviations.

The UAV is placed on a test stand to permit accurate attitude measurements. This test need not be extremely complicated however, and can usually be conducted with the vehicle on the ground. Very accurate, small, electronic angular measurement tools are available which allow alternate zero reference selection. Two such devices (calibrated) can be used to simultaneously measure air vehicle attitude in one axis and one control surface deflection. In addition, a device to stimulate the pitot-static system will be required. For a fixed wing conventional air vehicle the attitude control system test would include some or all of the following [1] - [3].

1) Level the air vehicle (this may require slight nose up to account for angle of attack in normal flight and wing incidence angle). 
2) Supply appropriate input to the pitot-static system to drive the elevator to neutral. This will vary according to the control laws for the specific air vehicle, but typically requires providing sufficient pitot pressure to match the airspeed report to the airspeed commanded in the ground control station (GCS).

3) Raise the nose 5 degrees and check for elevator deflection trailing edge down. The amount of travel can be verified if control laws are known. Verify GCS attitude display is in agreement. Repeat in 5-degree increments until maximum allowable elevator travel is reached.

4) Lower the nose 5 degrees and check for elevator deflection trailing edge up. The amount of travel can be verified if control laws are known. Verify GCS attitude display is in agreement. Repeat in 5-degree increments until maximum allowable elevator travel is reached.

5) Roll the air vehicle 5 degrees right and check for left aileron deflection, trailing edge up (or rudder trailing edge left if rudder is used for roll axis control). The amount of travel can be verified if control laws are known. Verify GCS attitude display is in agreement. Repeat in 5-degree increments until maximum allowable aileron travel is reached.

6) Roll the air vehicle 5 degrees left and check for left aileron deflection, trailing edge down (or rudder trailing edge right if rudder is used for roll axis control). The amount of travel can be verified if control laws are known. Verify GCS attitude display is in agreement. Repeat in 5-degree increments until maximum allowable aileron travel is reached.

7) While moving the air vehicle nose left, observe yaw rate display for correct direction, and rudder (if yaw or Dutch Roll damping is implemented) for deflection right.

The airspeed and altitude deviation response should also be checked. These will be dependent on control law implementation. In many cases, the altitude sensing system (usually static pressure, or radar) will drive the throttle actuator, and the airspeed system will drive elevator. Again, by inducing a difference between commanded and reported altitude and airspeed, the correct operating sense of the elevator and throttle can be verified (elevator trailing edge down for low reported airspeed, and throttle increase for low reported altitude). With fully defined control laws, the quantitative response can also be verified. These systems will in many cases have some interaction such as long term integrators if the difference between commanded and reported data exists for an extended period.

Results of the ground test [3] are shown in the following figures: Fig. 4b for the elevator response to speed variations, Fig. $4 \mathrm{c}$ for the altitude to throttle loop and Fig. 4a for heading control with lateral and directional commands. The maneuver consists in turning the UAV by 360 degrees while lifting it from the floor to maximum extension, the velocity is constant.
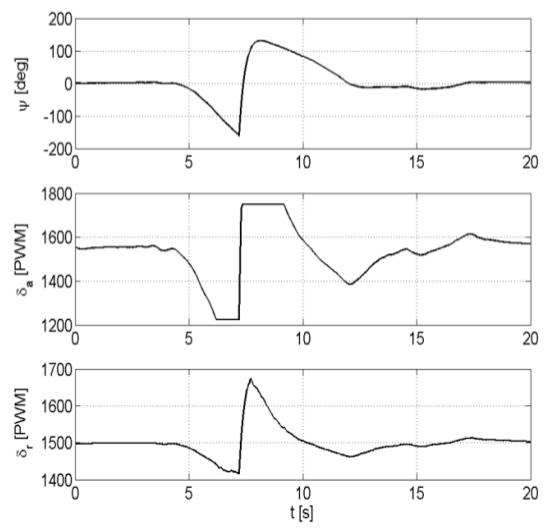

a
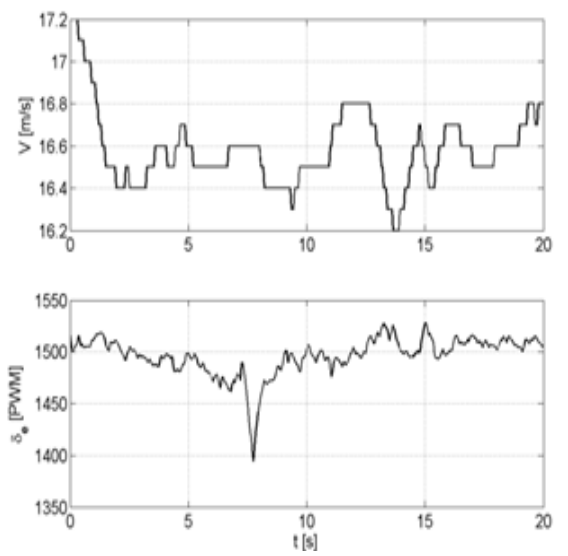
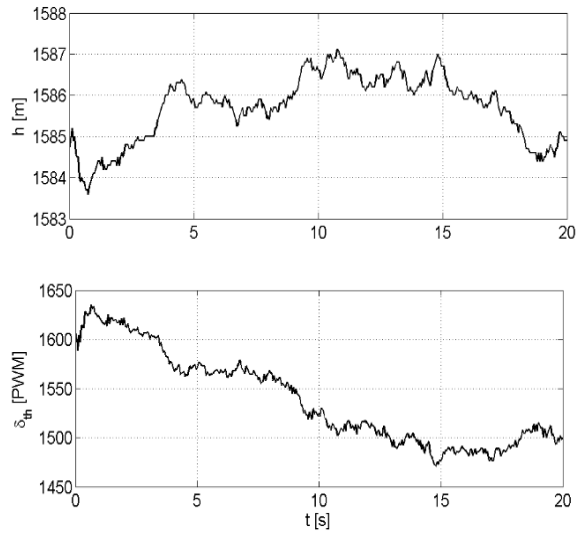

c

Fig. 4. UAV ground test results (from left to right $a, b, c$ ).

\section{CONCLUSION}

Dynamic ground testing system developed for critical UAV systems test, such as navigation and flight control system allow extended preflight check. Moreover, low cost of production for such testing system, which is comparable with stationary installations can bring UAV testing to the new quality level and decrease development, production and operation cost of modern unmanned aerial vehicles.

\section{REFERENCES}

[1] A. Pontzer, M. Lower, and J. Miller, "Unique Aspects of Flight Testing Unmanned Aircraft Systems," in NATO RTO AGARDograph 300, Flight Test Techniques Series, vol. 27, AC/323(SCI-105)TP/299, 2010.
[2] Naval Air Systems Command, Integrated Systems Evaluation, Experimentation and Test Department. Project Test Plan Policy and Guide for Testing Air Vehicles, Air Vehicle Weapons, and Air Vehicle Installed Systems. NAVAIRINST 3960.4B, 07 June 2005.

[3] Sartori D. Design, Implementation and Testing of Advanced Control Laws for Fixed-wing UAVs. PhD thesis, Politecnico di Torino, 2014.

[4] Jinyoung Suk, Jinhyung Kim. Ground Test and Evaluation of a Flight Control System for Unmanned Aerial Vehicles.. IJASS, vol. 5, no. 1, pp.57-63, May, 2004.

[5] M. A. A. Sanvido. Hardware-in-the-loop Simulation Framework. PhD thesis, Swiss Federal Institute of Technology, March 2002.

[6] R. Isermann, J. Schaffnit, and S. Sinsel. Hardware-in-the-loop simulation for the design and testing of engine-control systems. Control Engineering Practice, 7:643-653, 1999.

[7] R. Austin. Unmanned Aircraft Systems: UAVS Design, Development and Deployment. Wiley, 2010. 